\title{
Review of single particle dynamics for third generation light sources through frequency map analysis
}

\author{
L. Nadolski* and J. Laskar \\ Astronomie et Systèmes Dynamiques, IMC-CNRS UMR 8028 77, avenue Denfert Rochereau, 75014 Paris, France
}

(Received 17 March 2003; published 4 November 2003)

\begin{abstract}
Frequency map analysis [J. Laskar, Icarus 88, 266 (1990)] is used here to analyze the transverse dynamics of four third generation synchrotron light sources: the ALS, the ESRF, the SOLEIL project, and Super-ACO. Time variations of the betatron tunes give additional information for the global dynamics of the beam. The main resonances are revealed; a one-to-one correspondence between the configuration space and the frequency space can be performed. We stress that the frequency maps, and therefore the dynamics optimization, are highly sensitive to sextupolar strengths and vary in a large amount from one machine to another. The frequency maps can thus be used to characterize the different machines.
\end{abstract}

DOI: $10.1103 /$ PhysRevSTAB.6.114801

PACS numbers: 29.20.Dh, 29.27.Bd, 05.45.Ac

\section{INTRODUCTION}

Third generation light sources require very strong magnetic fields in order to achieve a small electron beam emittance and a high photon beam brightness together with a long Touschek lifetime. These strong magnetic fields can cause the motion of the electron to be strongly nonlinear and potentially unstable at large amplitudes resulting in a small injection efficiency and a short beam lifetime. As a result, the accelerator dynamists' work focuses on understanding the dynamics and adjusting the magnetic lattice in order to simultaneously achieve high brightness with a good injection rate and long lifetimes.

To achieve low emittances, third generation light sources are usually built with double bend achromat (DBA) lattice modules (ESRF, SOLEIL, Super-ACO), triple bend achromat (TBA) lattice modules (ALS), and theoretical minimum emittance lattice modules (SESAME). The lattice modules consist of different kinds of magnetic magnets distributed all around the ring, namely, dipoles for guiding electrons, quadrupoles for focusing, and sextupoles for correcting chromatic aberrations introduced by quadrupoles. Small emittances require strong quadrupole gradients which in turn require strong chromatic sextupoles used for correcting the linear chromaticities. They lead to geometric and nonlinear chromatic aberrations causing resonance excitation. In order not to lose the beam, other families of sextupoles are often used (except for the ALS) to minimize the resonance widths.

Therefore it is important to find a suitable arrangement of the sextupoles around the ring and to determine their strengths in order to minimize the nonlinearities leading

\footnotetext{
*Present address: Synchrotron SOLEIL, Saint-Aubin - BP48 91192 Gif-sur-Yvette CEDEX, France.

Email address: nadolski@synchrotron-soleil.fr
}

to unstable and even chaotic motions. Unfortunately, the prediction of resonance strengths is a difficult task. Generally whereas the chromatic sextupoles are adjusted to the desired chromaticities, the other sextupole families are used to reduce third order resonance widths $[1,2]$. The rule of thumb is that the resonances of lower orders are always the most dangerous. However many other resonances from low to high order are generated by sextupoles. A legitimate question for a real machine is to find the resonances that destroy the beam dynamics, to get an estimation of their strengths, and to correct them.

Throughout this article, the resonance identification is performed with Laskar's frequency map analysis (FMA). After a brief presentation of the method (Sec. II), applications are shown for four light sources, namely, the SOLEIL Project (Sec. III), the ESRF (Sec. IV), the Super-ACO ring (Sec. V), and the ALS (Sec. VI). For each machine, a so-called frequency map (FM) is plotted with its associated dynamic aperture (DA) either for the theoretical machine or for a running machine. Main resonances and effective dynamic aperture sizes are discussed. Then concluding remarks and perspectives are given in Sec. VII.

\section{FREQUENCY MAP ANALYSIS}

\section{A. Dynamical preliminaries}

In this work, only the single particle transverse dynamics was taken into account, which is reasonable given the very low longitudinal frequency, e.g., for SOLEIL, the longitudinal tune is $\nu_{s}=0.006$, whereas the horizontal tune is $\nu_{x}=18.28$. In order to get a complete picture of the dynamics, it is important to study both the on- and off-momentum dynamics. The on-momentum dynamics is what is relevant for understanding and optimizing the injection efficiency, and the off-momentum dynamics is relevant in understanding and optimizing the Touschek lifetime. However in our comparisons of various light 
sources, only the on-momentum dynamics is studied. For off-momentum studies, the reader is referred to Refs. [3,4].

The transverse dynamics is modeled by a $2+1$ degree of freedom system. The particle motion is described by the canonical coordinates $\left(x, y, l ; x^{\prime}, y^{\prime}, \delta\right)$ where $x$ and $y$ are the horizontal and vertical positions, $x^{\prime}, y^{\prime}$ the transverse normalized momenta; the relative trajectory lengthening with respect to the synchronous particle is denoted as $l$ and the relative momentum deviation $\delta$. For some magnetic field configurations, resonances can occur and induce nonlinearities and chaotic motions. A resonance appears for an integer linear combination of the transverse tunes $\nu_{x}, \nu_{y}$ and the revolution frequency $\nu=1$, i.e.,

$$
p \nu_{x}+q \nu_{y}+r=0,
$$

where $p, q$, and $r$ are integer numbers; $|p|+|q|$ is often called the resonance order. However here, we prefer to define the resonance order as the integer $|p|+|q|+|r|$ where only the fractional parts of the transverse tunes are considered in Eq. (1). Whenever this order is used, it will be denoted by $p: q: r$.

If the lattice is $M$ periodic, i.e., composed of $M$ identical sectors (this is the case for a perfect machine), the resonance condition is stronger: the dynamics is the same as that of a single sector with the revolution frequency $\nu=M$, and a resonance can now only occur if

$$
p \nu_{x}+q \nu_{y}+r \times M=0 .
$$

So light sources constructed on high periodicity enable us to suppress many resonances as illustrated by Fig. 1 . From now on, we shall talk about allowed and unallowed (by the ring periodicity) resonances. One should remember that an "unallowed resonance" of order $k=|p|+$ $|q|+|r|$ [Eq. (1)] can also be considered as an allowed resonance of higher order $|k| \times M$, with

$$
(p \times M) \nu_{x}+(q \times M) \nu_{y}+(r \times M)=0 .
$$
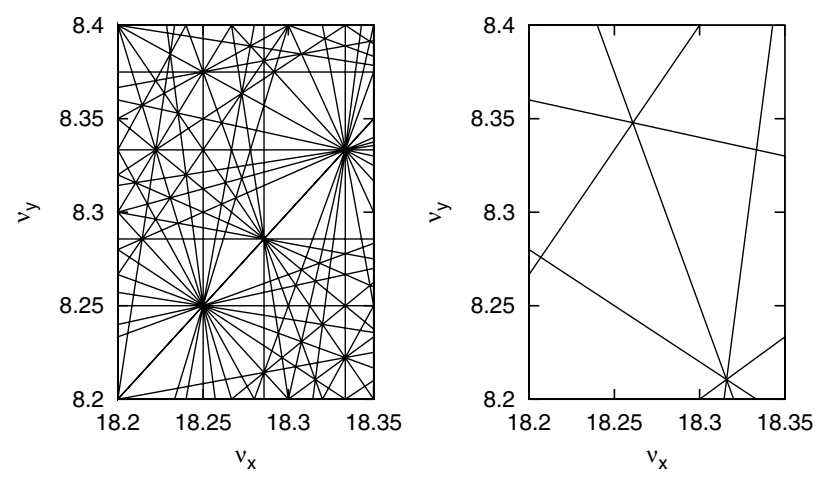

FIG. 1. Resonance diagram up to 8th order without (left panel) and with 12-fold periodicity (right panel).

\section{B. Method}

The study of the global dynamics of a beam is realized with the numerical FMA [5]. Briefly speaking, FMA constructs the so-called "frequency map" from the space of initial conditions to the tune space by searching for a quasiperiodic approximation of the transverse motion over a finite time span $T$. More precisely, two of the initial coordinates are fixed (here $x^{\prime}=y^{\prime}=0$ ) and $x, y$ are taken on a grid of initial values. Then for each set of initial conditions the test particle trajectory is numerically integrated and the discrete trajectory is recorded in a four-dimensional surface of section $x(t), y(t), x^{\prime}(t)$, and $y^{\prime}(t)$ over the time span $T$. By using a numerical algorithm based on some refined Fourier technique [6-9], we search for a quasiperiodic approximation of $z_{w}(t)=$ $w(t)+i w^{\prime}(t)$ of the form of a finite number of terms $N$ :

$$
z_{w}(t)=a_{w} e^{i \nu_{w} t}+\sum_{k=1}^{N} a_{\boldsymbol{m}_{k}} e^{i\left\langle\boldsymbol{m}_{k}, \boldsymbol{\nu}\right\rangle t},
$$

where $w=x, y, \boldsymbol{\nu}=\left(\nu_{x}, \nu_{y}, 1\right)$ is the fundamental frequency vector, $\boldsymbol{m}_{\boldsymbol{k}}=\left(m_{1 k}, m_{2 k}, m_{3 k}\right)$ is a multi-index, $\left\langle\boldsymbol{m}_{\boldsymbol{k}}, \boldsymbol{\nu}\right\rangle=m_{1 k} \nu_{x}+m_{2 k} \nu_{y}+m_{3 k}$, and the complex amplitude $a_{m_{k}}$ is ordered by decreasing magnitudes. For more details, the reader should refer to the article presented at the PAC 2003 [10], and references therein.

\section{Properties of the numerical frequency map $F^{T}$}

The frequency map $F^{T}$ is defined by

$$
\begin{gathered}
F^{T}: \mathbf{R}^{2} \rightarrow \mathbf{R}^{2}, \\
(x, y) \mapsto\left(\nu_{x}, \nu_{y}\right) .
\end{gathered}
$$

The transverse dynamics is described in the two- dimensional plane $\left(\nu_{x}, \nu_{y}\right)$ and is thus easy to plot. It should be noted that the image of $F^{T}$ in the frequency plane is largely independent of the initial momenta $\left(x_{0}^{\prime}, y_{0}^{\prime}\right)$ since the frequencies do not depend on phases [7]. The numerical FMA converges as $1 / T^{4}$ with a Hanning window $[8,10]$, which enables us to use only a small number of turns, typically 1000 in a running machine.

For a regular Kolmogorov-Arnold-Moser (KAM) solution, $F^{T}$ is invariant by time translation. In the other cases, the time variation of the tunes is related to the orbit diffusion, and its measure provides a stability criterion of the trajectory. Although for a 3 degree of freedom system KAM tori do not confine the trajectories over infinite time, the diffusion of the orbits decreases exponentially in the neighborhood of these KAM tori [11]. Thus a large number of regular orbits will act as an effective barrier to the diffusion in finite time (Fig. 2). Moreover, the frequency map $F^{T}$ is a regular function on the set of the KAM tori, and the study of the regularity of this map gives information about resonances and nonlinear behaviors $[8,10]$. 


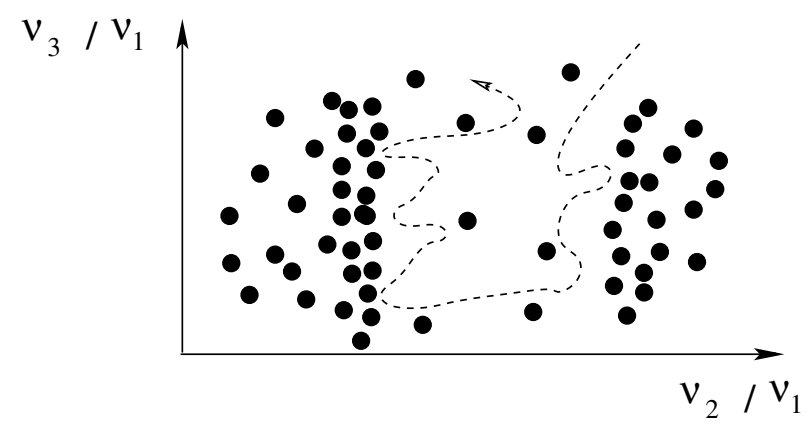

FIG. 2. Diffusion for a system of 3 degrees of freedom such as the transverse dynamics in an accelerator. Although not confined by regular trajectories (dots), the diffusion is nevertheless very slow in their neighborhood (from [12]).

\section{Steps for the study}

For practical use, a surface of section (or Poincaré surface) is chosen, typically at $s=0$ (the middle of a straight section of the accelerator). One looks at the return map with canonical transverse positions $(x, y)$ and momenta $\left(x^{\prime}, y^{\prime}\right)$. Given a set $\left(x_{0}, y_{0}, x_{0}^{\prime}=y_{0}^{\prime}=0\right)$ of initial conditions, the particle trajectory is numerically integrated over 2000 turns (the SOLEIL transverse and longitudinal damping times are, respectively, about 8000 and 4000 turns). For a surviving particle, the transverse tunes are computed with the FMA over the first 1000 turns, and one gets the tunes $\left(\nu_{x}^{(1)}, \nu_{y}^{(1)}\right)$.

As a stability index, we use the tune variation with time. The tunes $\left(\nu_{x}^{(2)}, \nu_{y}^{(2)}\right)$ are computed over the next 1000 turns, then the logarithm of the root mean square of tune difference gives a diffusion index $D$ :

$$
D=\log _{10}\left[\sqrt{\left(\nu_{x}^{(2)}-\nu_{x}^{(1)}\right)^{2}+\left(\nu_{y}^{(2)}-\nu_{y}^{(1)}\right)^{2}}\right] .
$$

This diffusion rate is coded by a color scale from blue, for very stable orbits, to red for unstable and chaotic ones. Because of the fast convergence of the method, the diffusion gives a good long-term stability criterion [13].

Our criterion for choosing the number of tracking turns is mainly determined in such a way that FMA converges fast enough in the vicinity of resonances where particle motion is not anymore quasiperiodic. For comparison, we also give the damping time expressed in turn number. The relationship between the number of tracking turns and damping times is not simple. Indeed damping processes are often beneficial for beam stability since particle amplitude gets smaller. Nevertheless through the damping process, particles can cross high nonlinear regions or resonances leading to amplitude increase and even particles lost. This is particularly true if the damping time is small with respect to the diffusion rate in the vicinity of the resonance (see, for example, Refs. $[4,14]$ ).

As the first nonlinear contribution of the amplitude to the frequency tune shift is quadratic, a square root step is chosen for the initial conditions. Consequently we have a more precise determination of the dynamics around the borders of the DA, i.e., in the vicinity of the resonances limiting the stability area, than at low amplitudes where the dynamics is known to be linear. Moreover we can obtain a one-to-one correspondence between the dynamic aperture and the tune space that gives also an insight of the DA inner structure. The next four sections are focused on the description of the light source dynamics through FMA.

\section{THE SOLEIL PROJECT}

\section{A. Lattice}

The SOLEIL project cell is based on a modified Chasman-Green structure [15] with distributed disper$\operatorname{sion}^{2}$. Table I sums up the main characteristics and the lattice functions are given by Fig. 3. The SOLEIL lattice is supposed perfect with a full fourfold periodicity; zero chromaticities and only on-momentum particles are tracked.

TABLE I. SOLEIL main parameters [15].

\begin{tabular}{lc}
\hline \hline Energy $(\mathrm{GeV})$ & 2.5 \\
Chromaticities $\xi_{x}, \xi_{y}$ & $0.0,0.0$ \\
Circumference $(\mathrm{m})$ & 337 \\
Emittance $\epsilon_{x}(\mathrm{~nm} \mathrm{rad})$ & 3 \\
Working point $\nu_{x}, \nu_{y}$ & $18.28,8.38$ \\
Physical aperture $(\mathrm{mm})$ & {$[-35,35] \times[-6.5,6.5]$} \\
\hline \hline
\end{tabular}

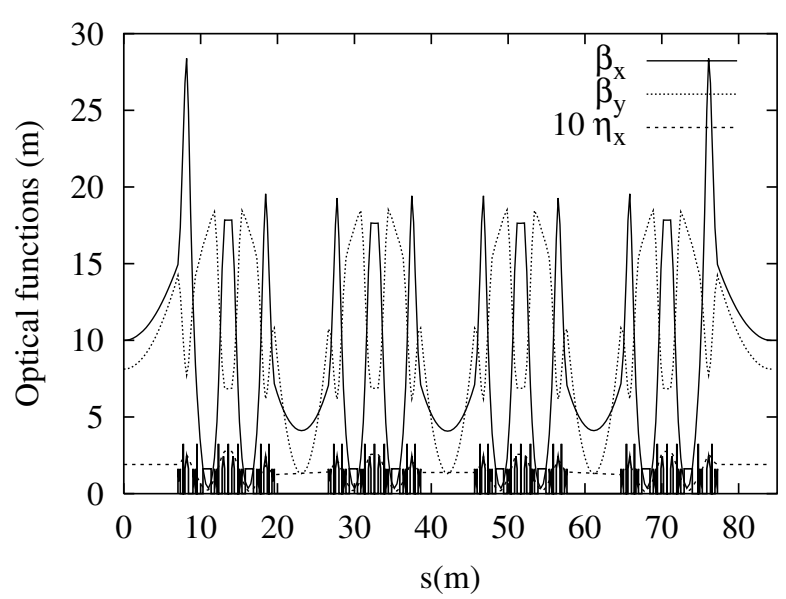

FIG. 3. Optical functions for one of the four SOLEIL superperiods composed of two double bend achromat cells.

\footnotetext{
${ }^{2}$ The reader should be aware that the SOLEIL lattice was modified in order to introduce eight additional new short traight sections since this study; the new lattice can be found in Ref. [16].
} 

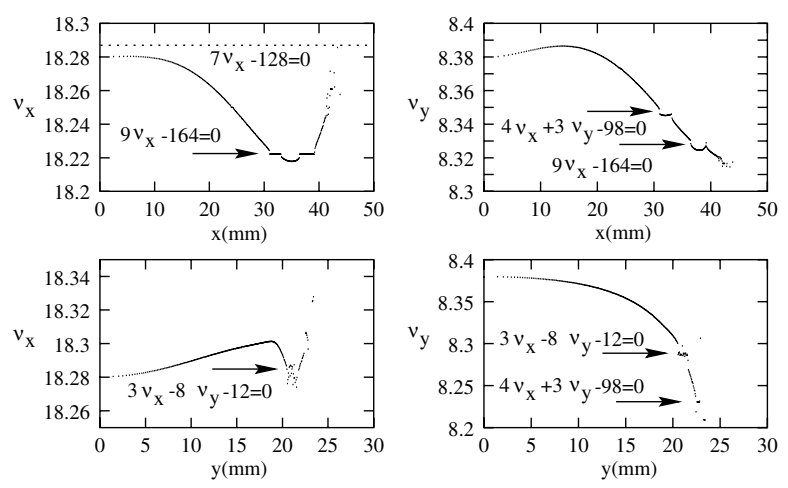

FIG. 4. SOLEIL first optics tune shifts with amplitude. For each case the fixed coordinate $x$ or $y$ is set to $1 \mu \mathrm{m}$.

\section{B. Dynamics}

Following the scheme described in Sec. II, the DAs and FMs are computed for three SOLEIL optics using the tracking code DESPOT [17]. All simulations are performed for single particles over 2000 turns, the last 1000 turns being devoted to the diffusion computation. The transverse damping time is $8.73 \mathrm{~ms}$, namely, 7771 turns; the longitudinal damping time is $4.35 \mathrm{~ms}$ or 3872 turns. Computations have been performed for a greater numbers of turns in order to check the result accuracies. Two low emittance optics were chosen and optimized [15].
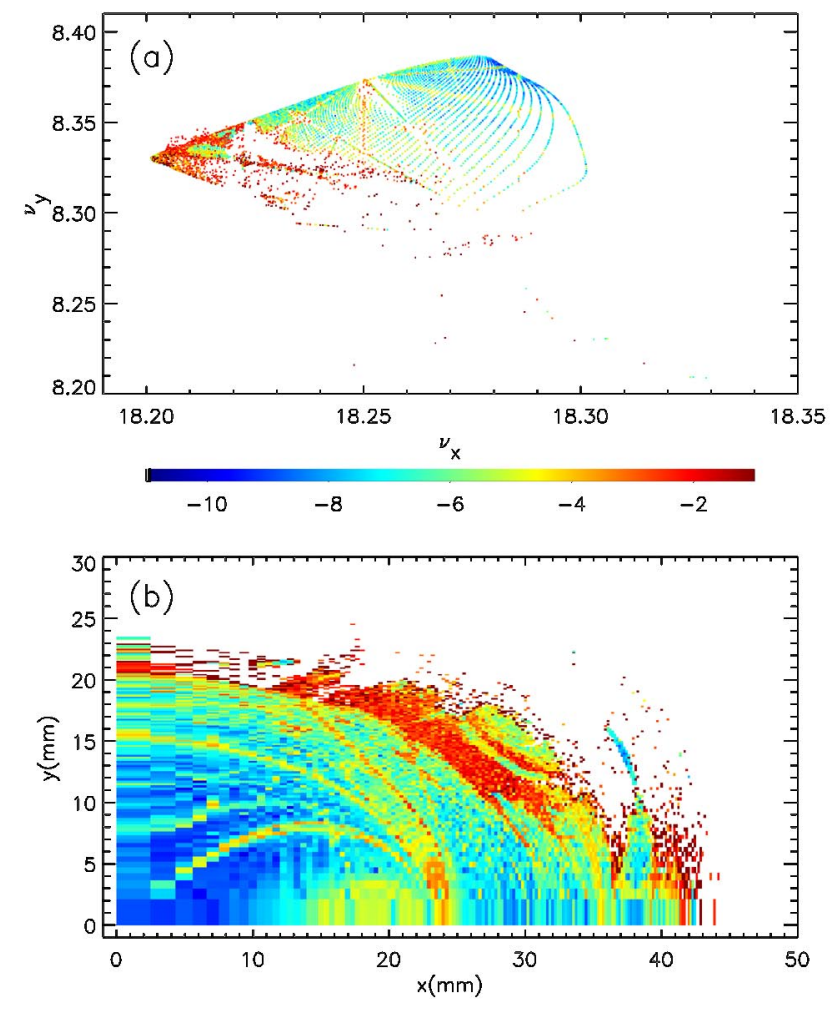

\section{First optics}

For the first optics, the working point is defined by the tunes $\left(\nu_{x}, \nu_{y}\right)=(18.28,8.38)$; the tune shifts with amplitude are plotted in Fig. 4. This optics has been optimized to constrain the dynamics between the two vertical resonance lines $7 \nu_{x}-4 \times 32=0$ (denoted in the figures, 7:0: -2$)$ and $9 \nu_{x}-4 \times 41=0(9: 0:-2)$.

From Fig. 5(b) one results in a large dynamic aperture, namely, $[0,40]_{y=0} \times[0,24]_{x=0} \mathrm{~mm}$. Yet these dimensions have to be slightly reduced to $35 \mathrm{~mm}$ in $x$ (taking into account the 9th-order resonance island) and $20 \mathrm{~mm}$ in $y$. The DA remains still larger than the physical aperture (see Table I).

Three kinds of areas can be identified on the corresponding frequency map [Fig. 5(a)], namely,

(i) regular areas where tune space points are regularly spaced with very low diffusion rates (blue-green color). The motion is a very regular betatron motion; see, for instance, the vicinity of the working point $\left(\nu_{x}, \nu_{y}\right)=$ $(18.28,8.38)$ (at low amplitudes the dynamics is obviously linear).

(ii) Resonances which are the straight lines of the rational slope, $p \nu_{x}+q \nu_{y}+r=0$. In their neighborhood there is either a lack of points if the resonance is crossed through a hyperbolic point or an accumulation of points for the elliptic case. The diffusion rate is larger: particles

(c)

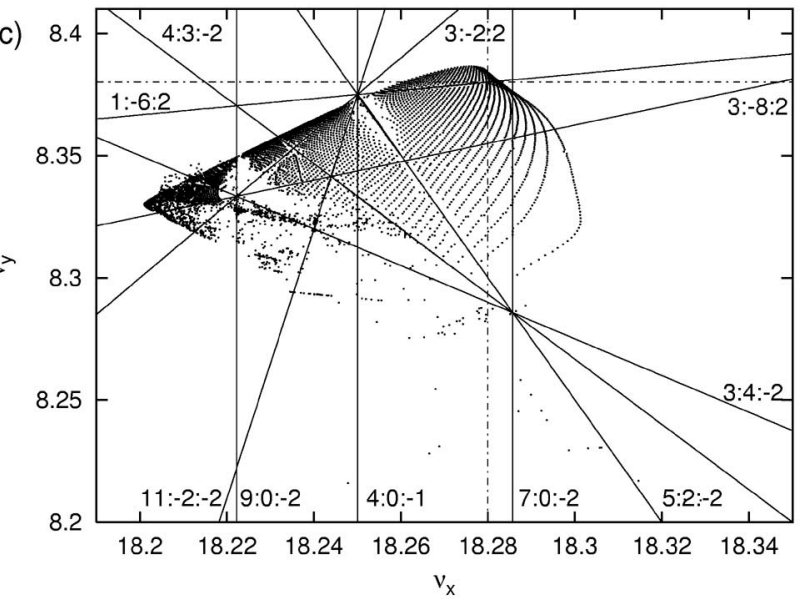

FIG. 5. (Color) SOLEIL first optics: Frequency map (a) and dynamic aperture (b) for the Poincaré surface $s=0$ ( $\beta_{x}=10 \mathrm{~m}$ and $\beta_{y}=8 \mathrm{~m}$ ). The large DA is marked by resonances, for instance, the green tongue at $x \approx 28$ mm corresponds to a 9 th-order resonance. The color scale indicates the orbit diffusion for regular (blue) to chaotic (red) motion. The main resonance networks are identified (c) and the working point is shown as a dashed line cross. 
tend to oscillate transversally to the resonance. For a region surrounded by regular motion the orbit diffusion is confined (cf. Fig. 2).

(iii) Irregular areas where all structure is lost with high diffusion rates (yellow to red color) which lead to nonlinear and even chaotic behaviors as this is the case at large amplitude and at the edges of the DA.

Globally the FM reveals a stable dynamics. Nevertheless several resonances are brought out. The 7 th-order coupling resonance, $5 \nu_{x}+2 \nu_{y}-4 \times 27=0$ $(5: 2:-2)$, reached for the horizontal amplitude $x=$ $24 \mathrm{~mm}$. In addition there is a resonance node just above, between this resonance, resonances $4 \nu_{x}-73=$ $0(4: 0:-1), 3 \nu_{x}-2 \nu_{y}-38=0(3:-2: 2), \nu_{x}-6 \nu_{y}+$ $4 \times 8=0 \quad(1:-6: 2), \quad$ and $11 \nu_{x}-2 \nu_{y}-4 \times 46=0$ (11: $-2: 2)$ [cf. Fig. 5(c)]. We can foresee that if this very node or the 7 th-order resonance were more excited, for instance by magnetic defaults, the fourfold symmetry of the ring would be broken and all the stable orbits beyond that resonance might become unstable. Then the horizontal DA would shrink horizontally to $25 \mathrm{~mm}$, to be smaller than the horizontal physical aperture.

Another interesting node affecting the dynamics is between the 7 th-order resonance $3 \nu_{x}+4 \nu_{y}-4 \times 22=$ $0 \quad(3: 4:-2)$, 9th-order resonance $9 \nu_{x}-4 \times 41=0$ (9:0: -2$)$, 11th-order $3 \nu_{x}-8 \nu_{y}+4 \times 3=0(3:-8: 2)$,
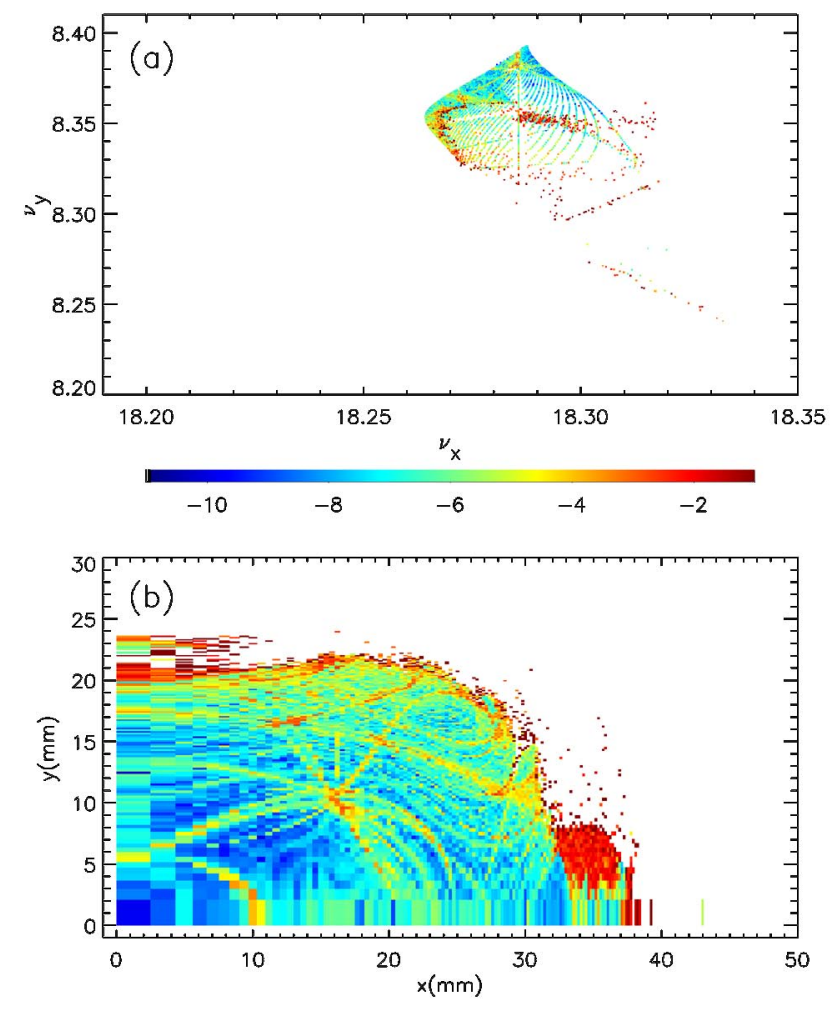

and 10th-order $6 \nu_{x}-4 \nu_{y}-4 \times 19=0(3:-2: 2)$. It is remarkable that not only low order resonances have to be taken into account, but higher order resonances such as 9 , 11, and 13 have non-negligible impact on the dynamics, especially at large amplitudes and at resonance nodes (for details, see [7]). Moreover, a one-to-one correspondence between the DA and the FM can be established, which reveals the localization of the resonances in the DA and particularly those restricting the DA. For instance, the small green stability tongue at $x \approx 38 \mathrm{~mm}$ in Fig. 5 (b) corresponds to 9th-order resonance islands in the FM [Fig. 5(a), $9 \nu_{x}-4 \times 41=0$ ].

\section{Modified first optics}

As it seems that the dynamics is mainly perturbed by the previous 9 th-order resonance and the associated node, while keeping the same working point, we modified the sextupolar strengths in order to avoid this resonance. Consequently, the amplitude tune shift slope at origin was changed from $\left(d \nu_{x} / d x^{2}\right)_{x=0}=300$ to 700 using the harmonic sextupoles. As seen in Fig. 6, the global beam dynamics becomes more regular for an almost same DA.

The main resonances are summarized in Fig. 6(c). Since the FM extension is smaller in the frequency space, less resonances are reached but in counterpart the FM is

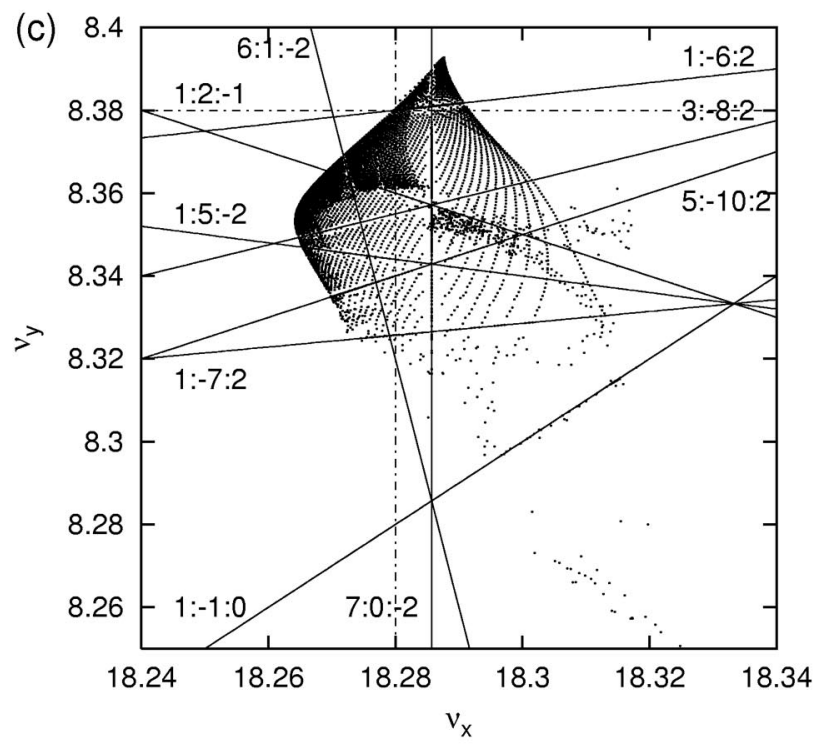

FIG. 6. (Color) SOLEIL modified first optics: Frequency map (a) and dynamic aperture (b) for the Poincaré surface $s=0\left(\beta_{x}=\right.$ $10 \mathrm{~m}$ and $\beta_{y}=8 \mathrm{~m}$ ). By avoiding the resonance 9:0: -2 , the global dynamics is significantly improved without any decrease of the DA. The FM is folded on itself (c), e.g., near the working point corresponding to the crossing of the two dashed lines. 

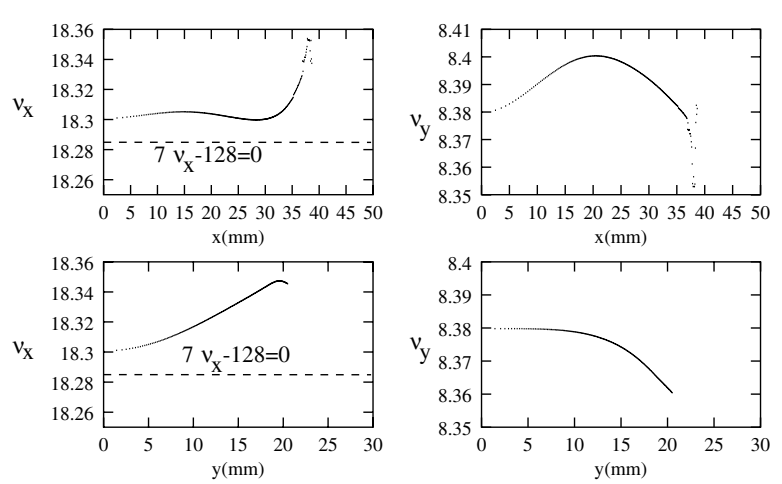

FIG. 7. Tune shifts with amplitude for the second optics of SOLEIL. For each case the fixed coordinate $x$ or $y$ is set to $1 \mu \mathrm{m}$. By using the harmonic sextupoles, the resonance of orders 7 and 9 are avoided (compare with Fig. 4).

more folded (compare Figs. 5 and 6). It is interesting to notice that, for very small modifications of sextupolar strengths, the FMs are quite different. For the same working point, the DA sizes are comparable but the transverse dynamics is largely changed.

\section{Second optics}

For the second optics, the new working point is $\left(\nu_{x}, \nu_{y}\right)=(18.30,8.38)$, in order that the horizontal tune never crosses the 7 th-order resonance $7 \nu_{x}-4 \times 32=0$ (see 7:0: -2 on Fig. 7 and compare with Fig. 4). The corresponding FM and DA are given by Fig. 8: the DA is slightly smaller than for the first case but more regular with much less diffusion than for the first optics (Fig. 5). The map is twice folded on itself: its extension is smaller in the frequency space, consequently the number of resonance encountered is rather low. On the other hand, this fold may lead to complicated dynamic features where rapid escaping directions may occur $[8,10]$.

\section{Summary on SOLEIL optics}

Disregarding the potential fold issue, among the two main optics chosen during the SOLEIL project, the second one seems to be the best concerning the beam stability, dynamic aperture size (always larger than the physical aperture), and with a low number of resonances encountered. However we see that FMA allows us to improve the first optics without significant loss of beam performance. This flexibility arises from the six harmonic sextupole families that can be used to optimize the dynamics without moving the working point. Fine adjustments and reduction of the resonances width may also be considered. These studies should be completed by integrating the off-momentum dynamics. We should stress the high sensitivity of the FM to the sextupole strengths.
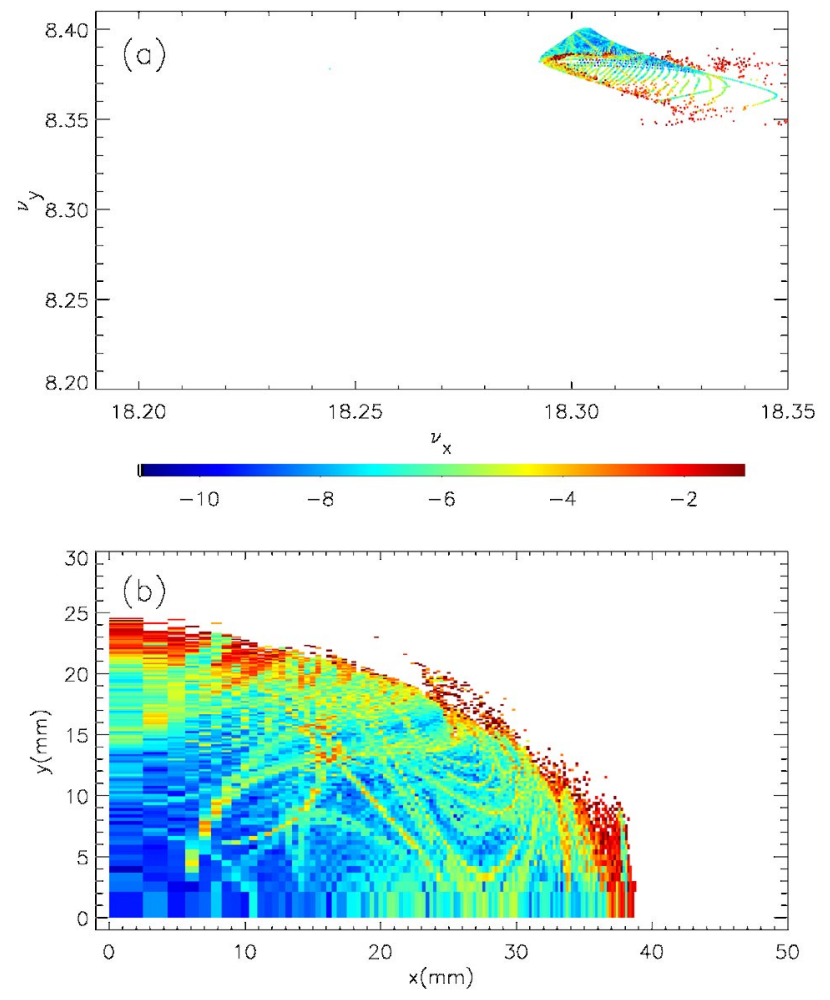

FIG. 8. (Color) SOLEIL second optics: Frequency map (a) and dynamic aperture (b) for the Poincaré surface $s=0\left(\beta_{x}=\right.$ $10 \mathrm{~m}$ and $\beta_{y}=8 \mathrm{~m}$ ).

\section{THE ESRF STORAGE RING}

\section{A. Lattice}

The European Synchrotron Radiation Facility (ESRF) lattice has a 16-fold periodicity [18]. This high periodicity allows us to reduce the number of allowed resonances. The storage ring lattice is built on a Chasman-Green structure with distributed dispersion, alternating low and high beta straight sections. The operation of the machine requires operating with large positive chromaticities. The main parameters and the lattice functions are summarized in Table II and Fig. 9. The measured momentum acceptance is about $\pm 2 \%$ and the injection is performed near the point $(x=-19 \mathrm{~mm}, y=0 \mathrm{~mm})$.

This study is restricted to the two sextupole sets which are currently used. Experimentally the second set seems better for high current per bucket. The tracking was

TABLE II. ESRF main parameters.

\begin{tabular}{lc}
\hline \hline Energy $(\mathrm{GeV})$ & 6 \\
Circumference $(\mathrm{m})$ & 844 \\
Emittance $\epsilon_{x}(\mathrm{~nm} \mathrm{rad})$ & 4 \\
Chromaticities $\xi_{x}, \xi_{y}$ & $0.1,0.4$ \\
Working point $\nu_{x}, \nu_{y}$ & $36.44,14.39$ \\
Physical aperture $(\mathrm{mm})$ & {$[-19,35] \times[-4,4]$} \\
\hline \hline
\end{tabular}




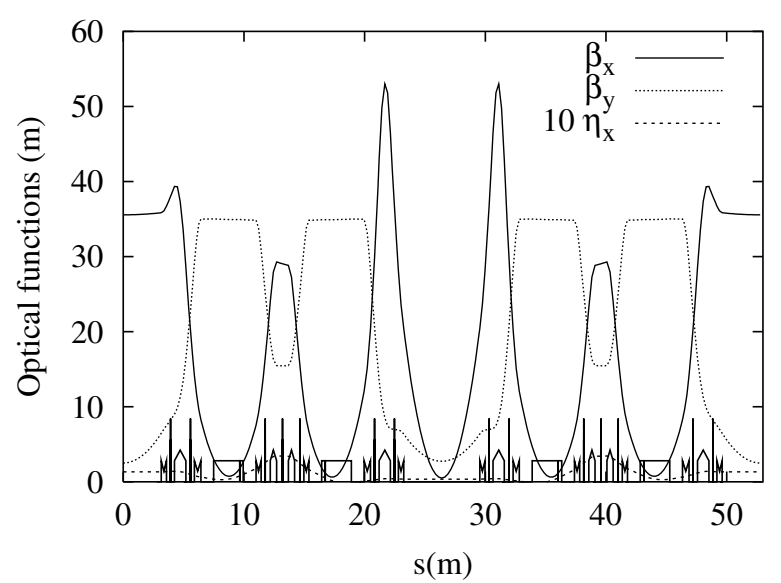

FIG. 9. Lattice functions for one of the 12 ESRF superperiods composed of two TBA cells.

performed twice over 1000 turns of a machine composed of only one superperiod. The ESRF damping time is about 2000 turns. The symplectic integrator LIE4 from the MAD program [19] was used as a tracking code for on-
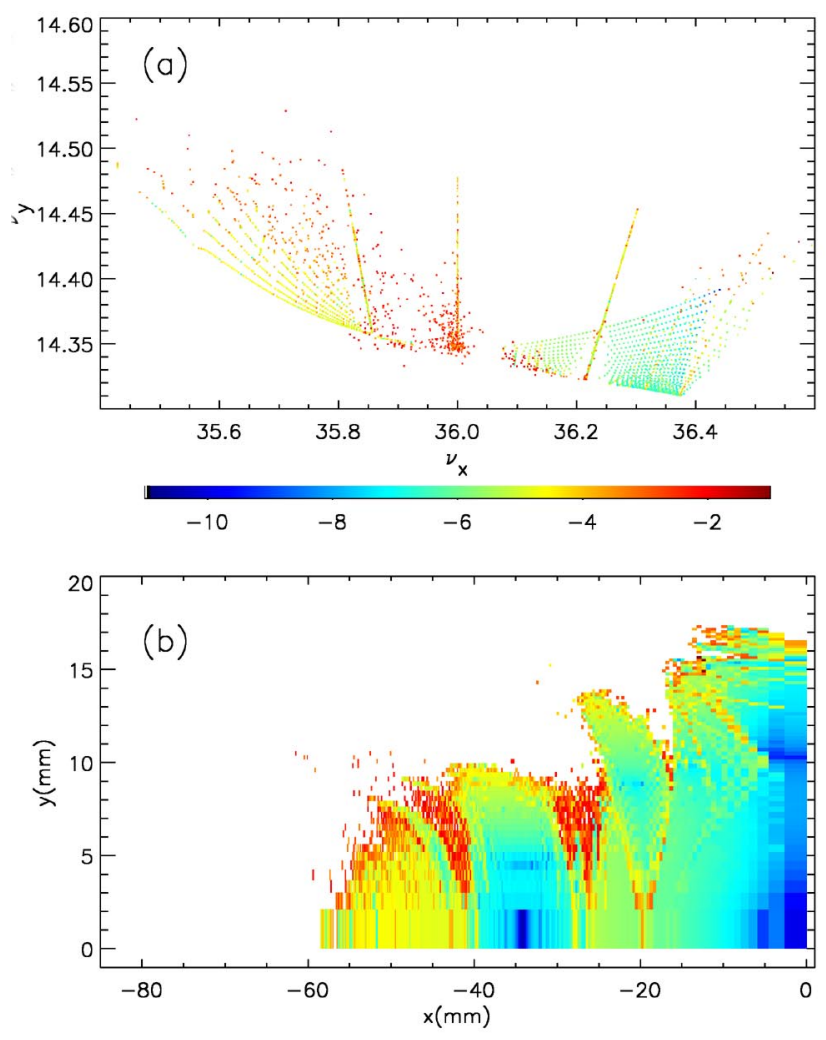

momentum particles. A characterization of both sets is given using FMA which gives some insight into the dynamical behavior.

\section{B. Dynamics}

\section{First sextupolar set}

The Figs. 10(b) and 10(d) exhibit the ESRF DA; at first glance, it seems very large, asymmetrical, and strongly affected by resonances: $[-56,42]_{y=0} \times[-16,16]_{x=0} \mathrm{~mm}$ $\left(\beta_{x}=36 \mathrm{~m}\right.$ and $\left.\beta_{y}=2.5 \mathrm{~m}\right)$. Nevertheless once again these dimensions have to be reduced. Indeed a more accurate analysis of its inner structure reveals numerous resonance islands inflating it artificially. The reading of an ESRF FM [Fig. 10(a)] is more complicated than the SOLEIL one since the map is strongly folded. The frequency map can be divided into two parts: (i) an upper part: around the working point, extending up to $20 \mathrm{~mm}$ in $x$ (limited by the integer resonance $\nu_{x}-36=0$ ), and $7 \mathrm{~mm}$ in $y$ with very low diffusion. However this part is not free of resonances: the 5th-order resonance
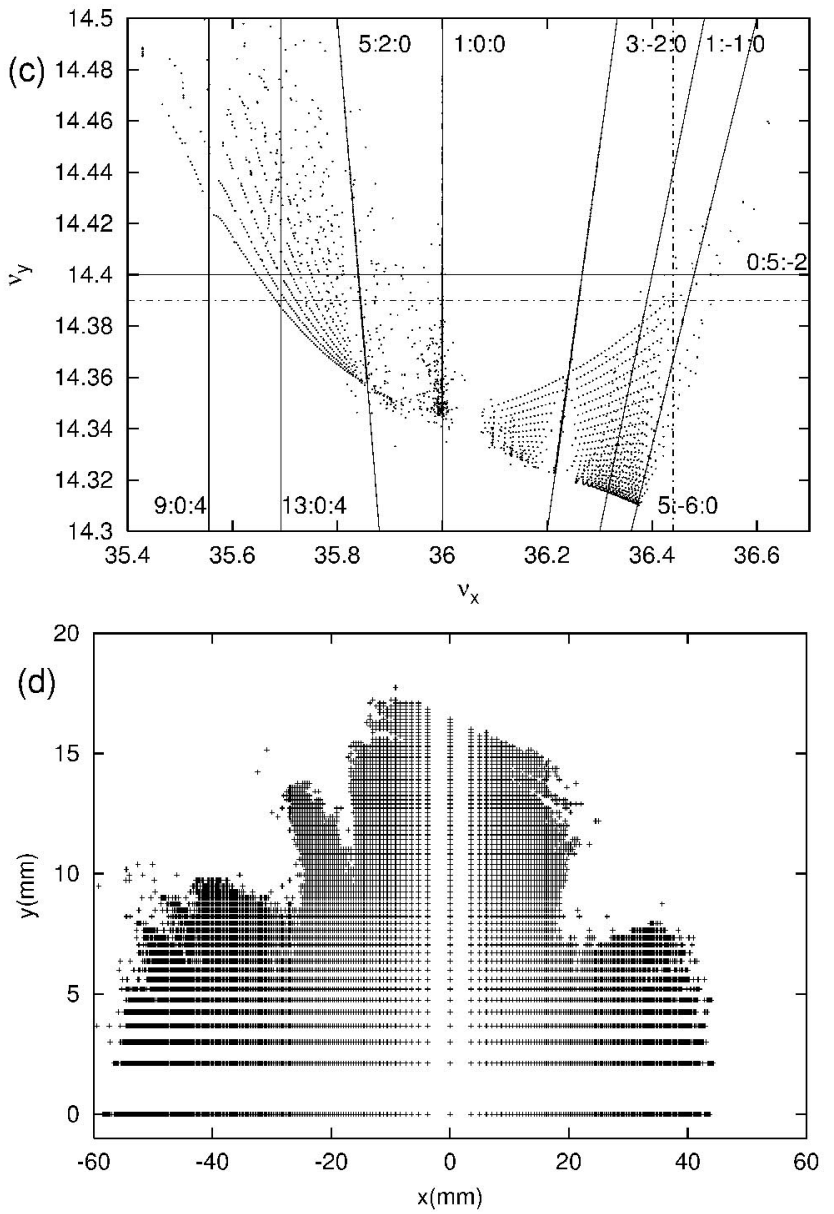

FIG. 10. (Color) ESRF frequency map (a) and dynamic aperture (b) for the first sextupole set computed for the Poincaré surface $s=0\left(\beta_{x}=35.6 \mathrm{~m}\right.$ and $\left.\beta_{y}=2.5 \mathrm{~m}\right)$. Although very large, the DA is irregular and distorted by many resonances and dominated by the coupling resonance 3: $-2: 0$ and the integer resonance $\nu_{x}-36=0$. Resonance network (c) and full dynamic aperture (d). The working point is shown by the dashed line cross. 
$3 \nu_{x}-2 \nu_{y}-5 \times 16=0(3:-2: 0)$ is reached for $x=$ $17 \mathrm{~mm}$ in the configuration space $(x=-20 \mathrm{~mm}$ for the negative DA). (b) A lower part: where the tune shift is greater than the unity. Very few regular areas last and the diffusion index is higher. Many resonances and nodes of resonances appear from low orders, 4, 5, to high orders, $8, \ldots, 16$ [see Fig. 10(c)]. For instance the wide resonance $\nu_{x}-36=0$ traps all particles for $x \in[-41,-28]$ or $x \in$ $[20,30] \mathrm{mm}$. As the integer resonance will never be crossed without loss of the beam and significant reduction of beam performance, we can derive a more precise DA as $[-27,21]_{y=0} \times[-7,7]_{x=0} \mathrm{~mm}$, that is to say a reduction of a factor 2 in both transverse degrees of freedom.

In addition tune shift with momentum allows us to assert that integer resonance $\nu_{x}-36=0$ (1:0:0) will be reached sooner for off-momentum particles, so the offmomentum DA will be even much smaller. Of course taking into account magnetic errors for the lattice model, the periodicity will be broken, resonance widths will become larger, and stability areas in the frequency space smaller. The dynamics might then be driven by the 5th-
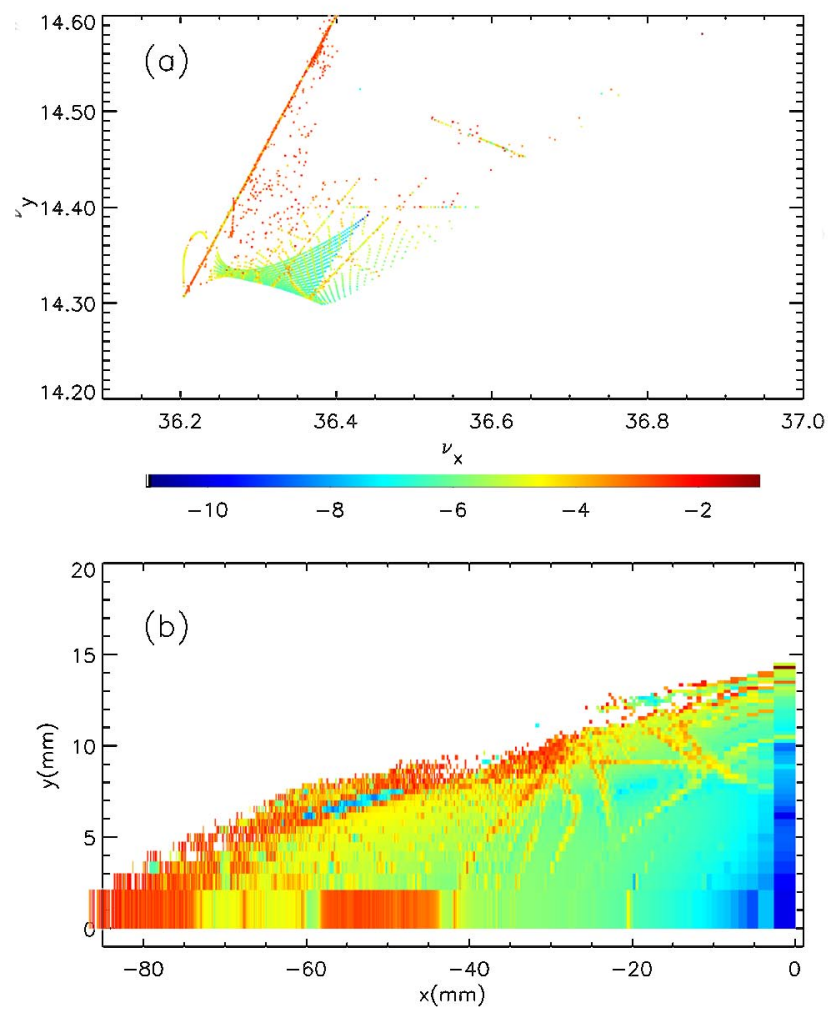

order coupled resonance $3 \nu_{x}-2 \nu_{y}-5 \times 16=0$ (3: 2:0) as well as for on- and off-momentum particles. As a result, the Touschek lifetime is probably limited by the transverse and the off-momentum dynamics.

\section{Second sextupolar set}

For this sextupolar set, the working point remains the same; the first term in the tune shift with amplitude $\left(d \nu_{x} / d x^{2}\right)_{x=0}$ is decreased from -23000 to -8400 and yet the beam dynamics is fully modified (Fig. 11).

The DA appears to be very large: $[-80,45]_{y=0} \times$ $[-15,15]_{x=0}$ but is still overestimated. As previously for an easy reading, the FM can be split into two parts [Fig. 11(a)]: the upper part extending up to $[-30,20]_{y=0} \times[-8,8]_{x=0} \mathrm{~mm}$, with regular, almost free of resonances, and which is characterized by very little diffusion; and the lower part with numerous resonance whose orders are summarized in Fig. 11(c). Although the integer resonance $\nu_{x}-36=0$ is never reached, the 5 th-order resonance $3 \nu_{x}-2 \nu_{y}-5 \times 16=$
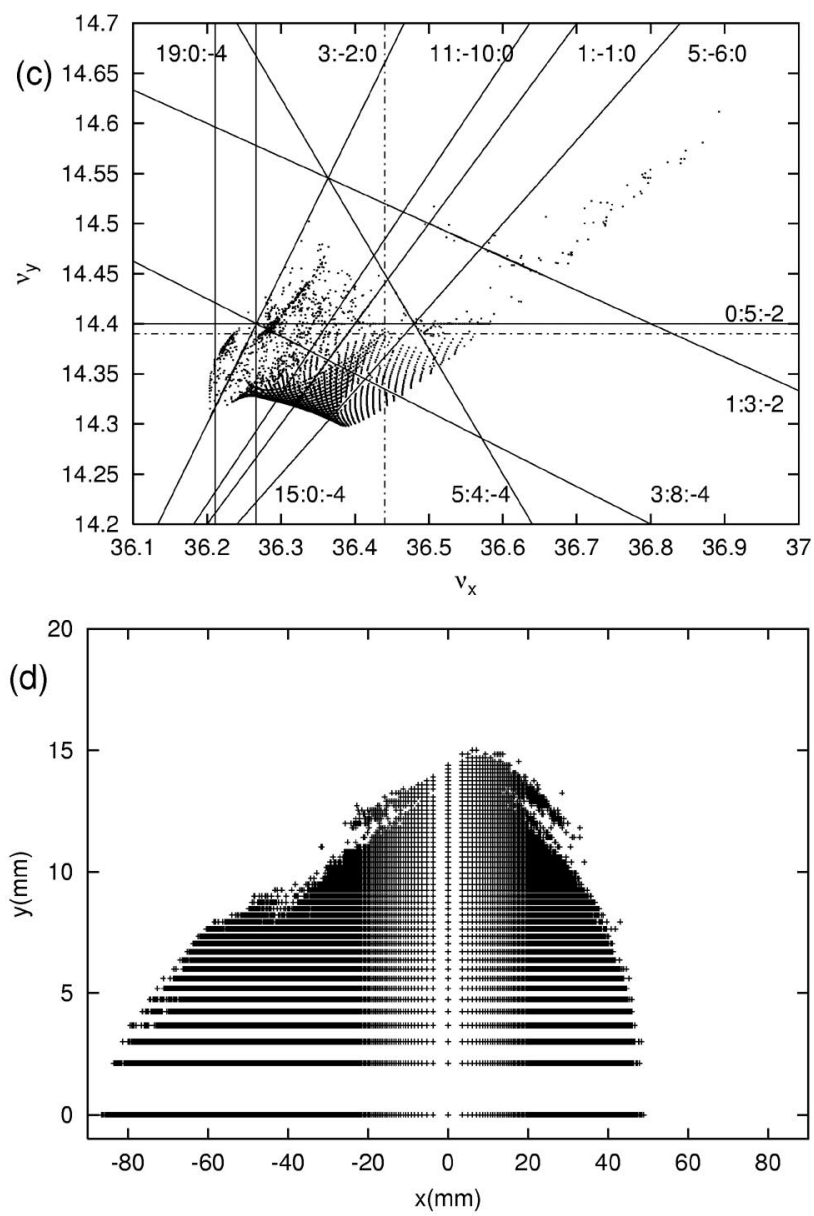

FIG. 11. (Color) ESRF frequency map (a) and dynamic aperture (b) for the second sextupole set computed for the Poincaré surface $s=0\left(\beta_{x}=35.6 \mathrm{~m}\right.$ and $\left.\beta_{y}=2.5 \mathrm{~m}\right)$. Resonance identification (c) and full dynamics aperture (d): The working point is shown as a dashed line cross. The main resonance 3: $-2: 0$ is now reached at a larger amplitude. As a result, the dynamics is globally improved. 
0 is already strong for a perfect lattice since it traps all particles for $x \in[-74,-42]$ or $x \in[32,35] \mathrm{mm}$.

On the whole the dynamics for this set is less complicated and more stable. The tune shift with amplitude is smaller. Consequently the 5 th-order resonance $3 \nu_{x}-$ $2 \nu_{y}-5 \times 16=0$ is reached for a larger amplitude, $x=$ $-42 \mathrm{~mm}$ ( $-19 \mathrm{~mm}$ for the first sextupolar set).

\section{Summary on the ESRF optics}

Looking at the ESRF FMs, the first set seems the most harmful for the dynamics. This feature becomes even more relevant when the current per bucket increases since larger on- and off-momentum dynamics apertures are then required. In an intuitive way, we can also understand why the performance was quite disappointing in terms of lifetime and injection efficiency when the ESRF ring was started. The nominal working point was defined by the tunes $\nu_{x}=36.2$ and $\nu_{y}=14.3$. The working point was thus closer to the vertical integer resonance. The DA shrunk and was characterized by a global high diffusion rate. To this end, new numerical simulations were recently performed for a more realistic lattice taking into account focusing and coupling errors; first experimental frequency maps were also obtained showing excitation of mainly 5th- and 10th-order resonances [20].

\section{SUPER-ACO}

\section{A. Lattice}

The Super-ACO ring [21] can be associated with the third generation light sources in spite of its large horizontal emittance. Indeed it was the first machine whose straight sections were fully equipped with insertion devices. Its lattice is designed on a DBA structure with a fourfold periodicity. Its main characteristics and lattice functions are given by Table III and Fig. 12. The damping time is 75000 turns, and the longitudinal tune is $\nu_{s}=$ 0.0034 . The particle tracking was performed with the MAD program over twice 1000 turns. Because of its short circumference, Super-ACO dipoles are modeled with fringe fields. In addition, each quadrupole is a combined magnet with a sextupole component and a strong 10-poles systematic magnetic default.

TABLE III. Super-ACO main parameters.

\begin{tabular}{lc}
\hline \hline Energy $(\mathrm{MeV})$ & 800 \\
Circumference $(\mathrm{m})$ & 72 \\
Emittance $\epsilon_{x}(\mathrm{~nm}$ rad $)$ & 38 \\
Chromaticities $\xi_{x}, \xi_{y}$ & $0.0,0.0$ \\
Working point $\nu_{x}, \nu_{y}$ & $4.72,1.70$ \\
Physical aperture $(\mathrm{mm})$ & {$[-20,60] \times[-15,15]$} \\
\hline \hline
\end{tabular}

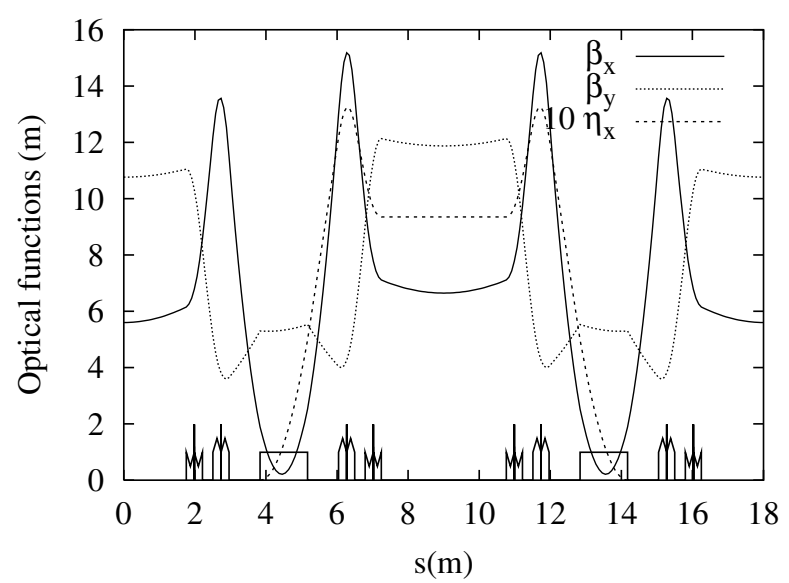

FIG. 12. One of the four Super-ACO double bend achromat cells.

\section{B. Dynamics}

The Super-ACO tune shifts with amplitude are given by Fig. 13. Several resonances are outlined. The DA dimensions are $[-50,50]_{y=0} \times[-40,40]_{x=0} \mathrm{~mm}$ [Figs. 14(b) and 14(d)], but are reduced to $\pm 20 \mathrm{~mm}$ in the vertical plane after carefully analyzing the beam dynamics through resonance identification and orbit diffusion. Most particles with an initial vertical position $y>$ $23 \mathrm{~mm}(x=0 \mathrm{~mm})$ are trapped in the islands of the strongly excited 3rd-order resonance $\nu_{x}+2 \nu_{y}-2 \times 4=$ $0(1: 2:-2)$. For $y<0$, this island does not appear as the section cuts through a hyperbolic fixed point, hence the strong DA asymmetry. Its dimensions become then $[-50,50]_{y=0} \times[-23,23]_{x=0} \mathrm{~mm}$.

The FM sweeps a large region of the frequency space down to $\nu_{y}=1.51$ [Fig. 14(a)]. Only the part above the 3rd-order coupled resonance is shown. The part beyond is discarded since this resonance will never be crossed, except with a partial or total beam loss for a running machine. Globally the beam dynamics is mainly dominated by this coupled resonance $\nu_{x}+2 \nu_{y}-2 \times 4=0$ $(1: 2:-2)$. In its vicinity, a particle rapidly escapes to unbounded motions. Other particles are trapped in the resonance islands. Resonances from all order are given in Fig. 14(c). On the other hand, around the working point $\left(\nu_{x}, \nu_{y}\right)=(4.72,1.70)$, the dynamics is very regular with a low diffusion rate and no visible resonances. Nevertheless we should point out that the FM is strongly folded in the area $\nu_{x} \approx 4.7075$ [see Figs. 14(a) and 14(c)]. The motion is highly unstable beyond, namely, for horizontal positions $|x|>30 \mathrm{~mm}$.

\section{THE ALS}

\section{A. Lattice}

The Advanced Light Source (ALS) [22] is a 12-fold periodic ring. The lattice is a TBA mirrored about its center (Fig. 15). The only nonlinearities are introduced by 

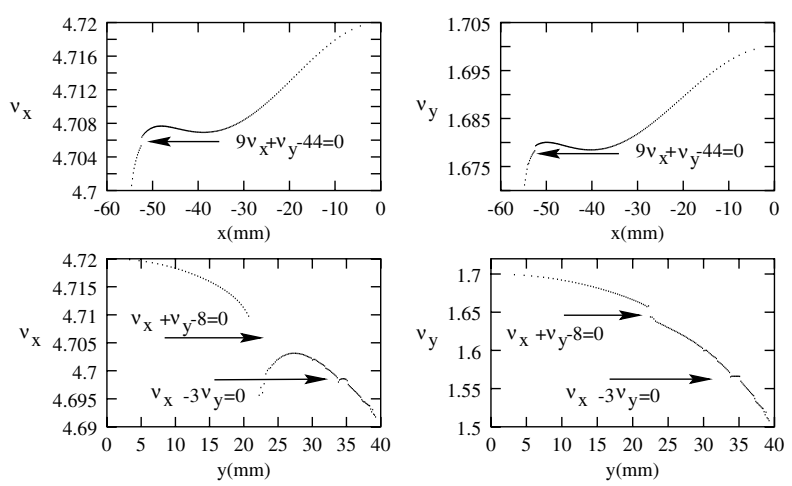

FIG. 13. Super-ACO tune shifts with amplitude. For each case the fixed coordinate $x$ or $y$ is set to $1 \mu \mathrm{m}$.

the two sextupole families using for correcting the chromaticities slightly overcompensated to $\left(\xi_{x}, \xi_{y}\right)=$ $(0.5,1.0)$ (cf. Table IV). For the tracking, the DESPOT computer code was used over twice 1000 turns, i.e., about
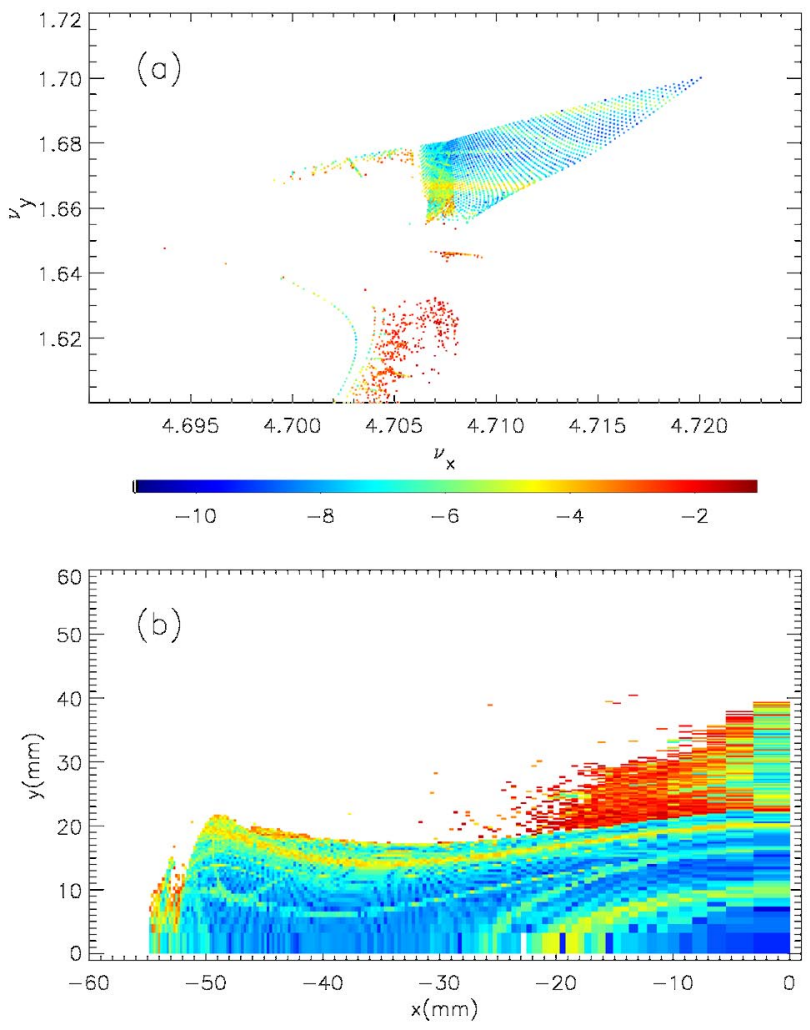

one-twelfth of the ring damping time. The actual working point is very close to the one proposed in 1996 after a first application of FMA [23]. The dynamics of the ALS has already been extensively studied through FMA $[4,13,23,24]$, and we present it here for comparison with the other machines.

\section{B. Dynamics}

\section{Perfect lattice}

At first, a perfect ALS lattice is studied. The ring has high periodicity: its 12 identical sectors enable us to strongly reduce the number of allowed resonances in the frequency space. It follows that the DA is really large: $[0,22]_{y=0} \times[0,10]_{x=0} \mathrm{~mm}$, whereas the experimental horizontal DA is smaller, around $11 \mathrm{~mm}$ [Fig. 16(b)].

On the contrary to the other studied machines, the ALS FM is very simple and regular with no fold: the working point $\left(\nu_{x}, \nu_{y}\right)=(14.25,8.18)$ is the upper right corner of
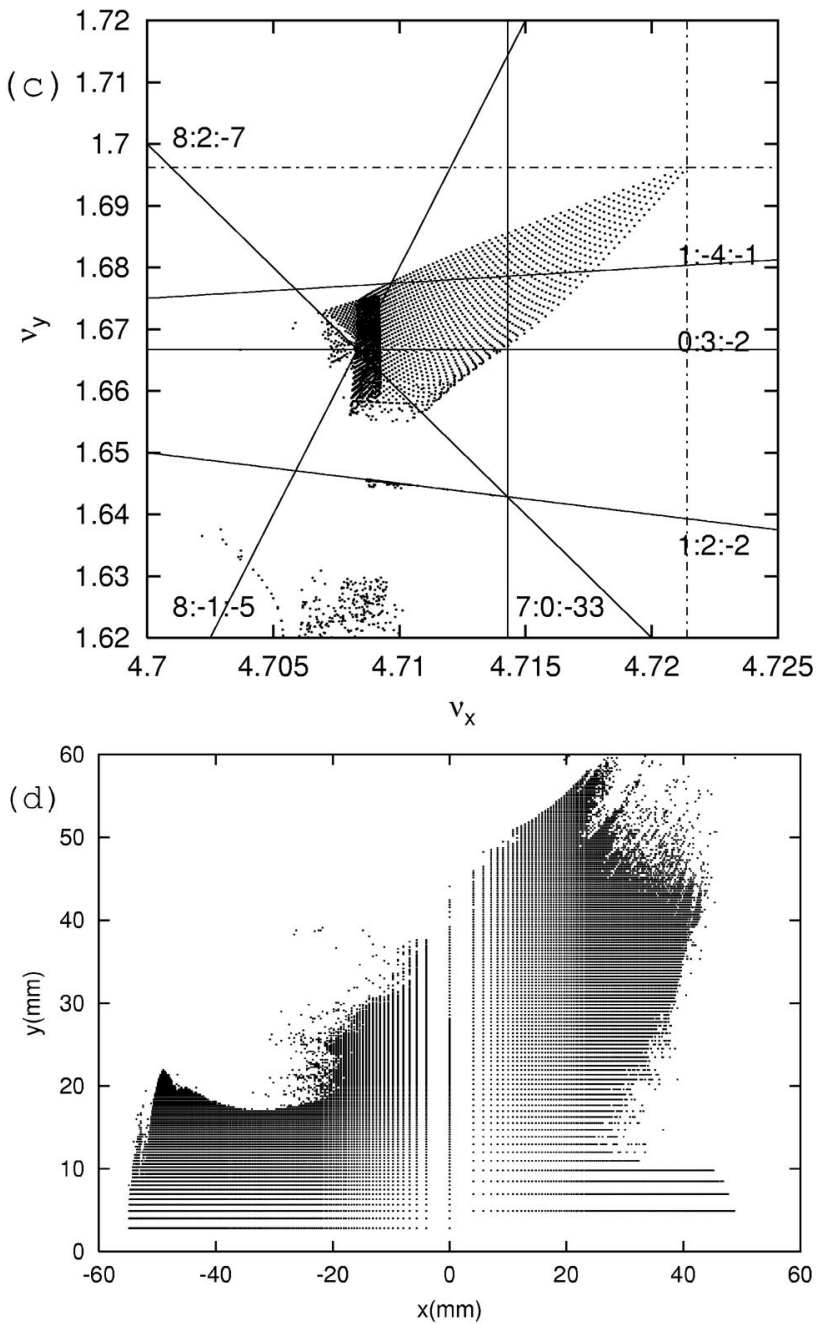

FIG. 14. (Color) Super-ACO frequency map (a) and dynamic aperture (b): bare lattice for the Poincaré surface $s=0\left(\beta_{x}=5.6 \mathrm{~m}\right.$ and $\beta_{y}=10.8 \mathrm{~m}$ ). The lower part of the FM is not show since it is useless physics wise. Resonance network (c) and full dynamic aperture (d). The working point is shown as a dashed line cross. 


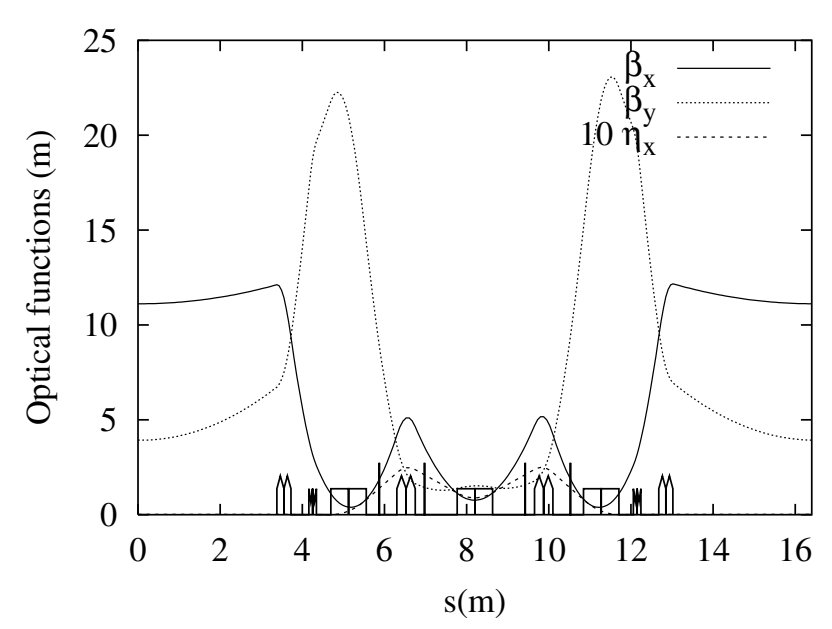

FIG. 15. One of the 12 ALS TBA cells.

the FM. The upper side (respectively, lower side) corresponds to the tune shift with the vertical (horizontal) amplitude. The two strongest resonances are the integer resonance $\nu_{y}-8=0$ and the coupling resonance $\nu_{x}-$ $\nu_{y}-6=0(1:-1: 0)$ [Fig. 16(c)]. Far from the working point the resonances overlap, inducing chaotic zones with a large diffusion index corresponding to strong nonlinear behavior and limiting the DA [Fig. 16(a)].
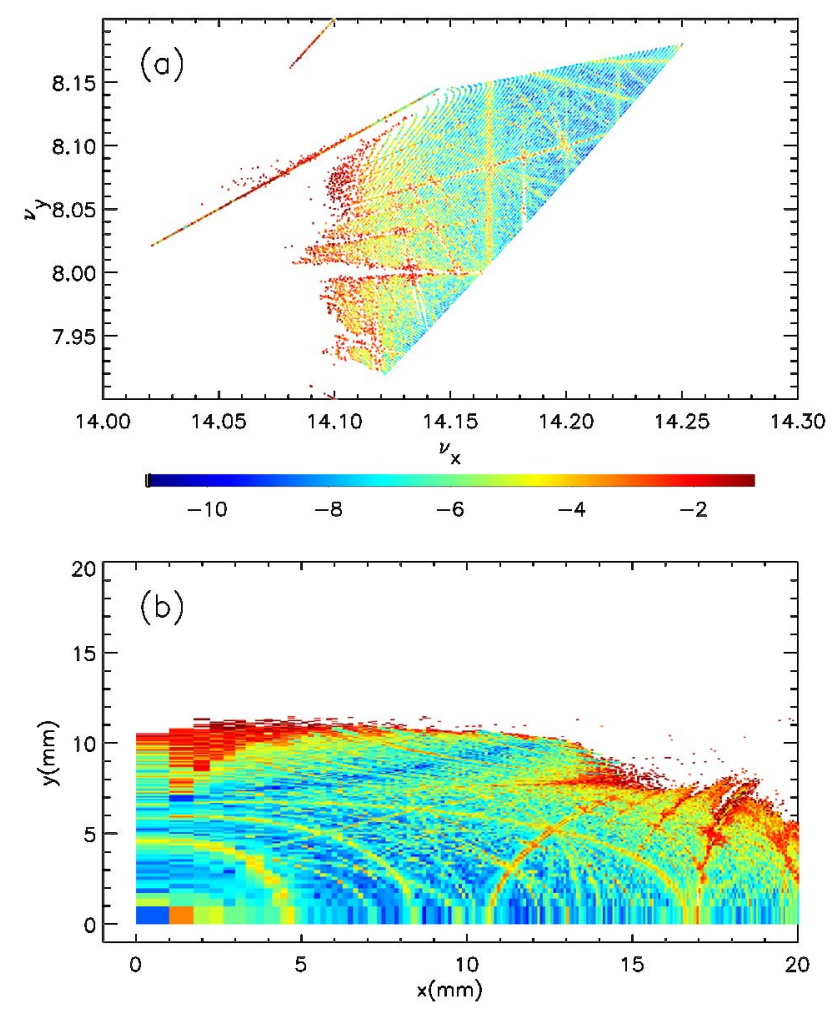

TABLE IV. Nominal ALS parameters.

\begin{tabular}{lc}
\hline \hline Energy $(\mathrm{GeV})$ & 1.9 \\
Circumference $(\mathrm{m})$ & 196 \\
Emittance $\epsilon_{x}(\mathrm{~nm}$ rad $)$ & 5.5 \\
Chromaticities $\xi_{x}, \xi_{y}$ & $0.5,1.0$ \\
Working point $\nu_{x}, \nu_{y}$ & $14.25,8.18$ \\
Physical aperture $(\mathrm{mm})$ & {$[-25,25] \times[-2.5,2.5]$} \\
\hline \hline
\end{tabular}

\section{More realistic lattice}

A more realistic lattice of the ALS is now used by including both normal and skew quadrupole errors obtained by analyzing the linear orbit response matrix of the ring [25] with the LOCO code [26]. Knowing the lattice quadrupole errors, it is possible to restore the symmetry. As a measurement of the goodness of the correction, the beta beating is reduced to the order of $1 \%$. The average normal quadrupole error is $0.36 \% \mathrm{rms}$ and $0.2 \% \mathrm{rms}$ in focusing and defocusing magnets. Skew quadrupole errors are adjusted in such a way that the effective coupling becomes 1\%. The new FM and DA are plotted on Fig. 17 (to compare with Fig. 16): the global dynamics is fully modified, the 12 -fold periodicity is broken, so the resonance condition becomes weaker. As a consequence the diffusion is higher and the resonances wider; the integer

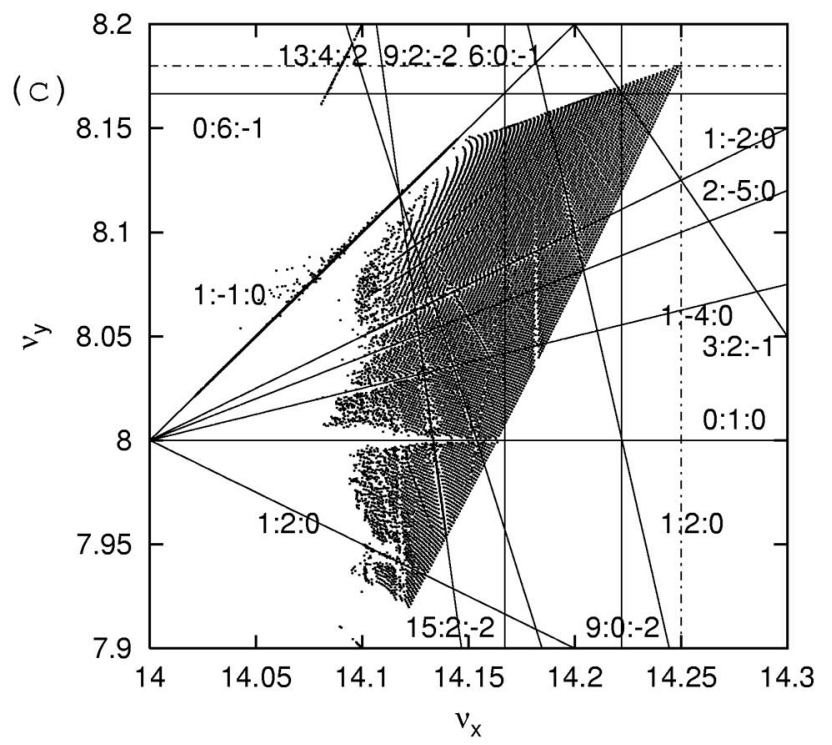

FIG. 16. (Color) ALS frequency map (a) and dynamic aperture (b): bare lattice at the location $s=0\left(\beta_{x}=11.3 \mathrm{~m}\right.$ and $\beta_{y}=$ $4.0 \mathrm{~m}$ ). The orbit diffusion shows clearly the resonance network. Resonance chart (c): The working point is shown as a dashed line cross. 

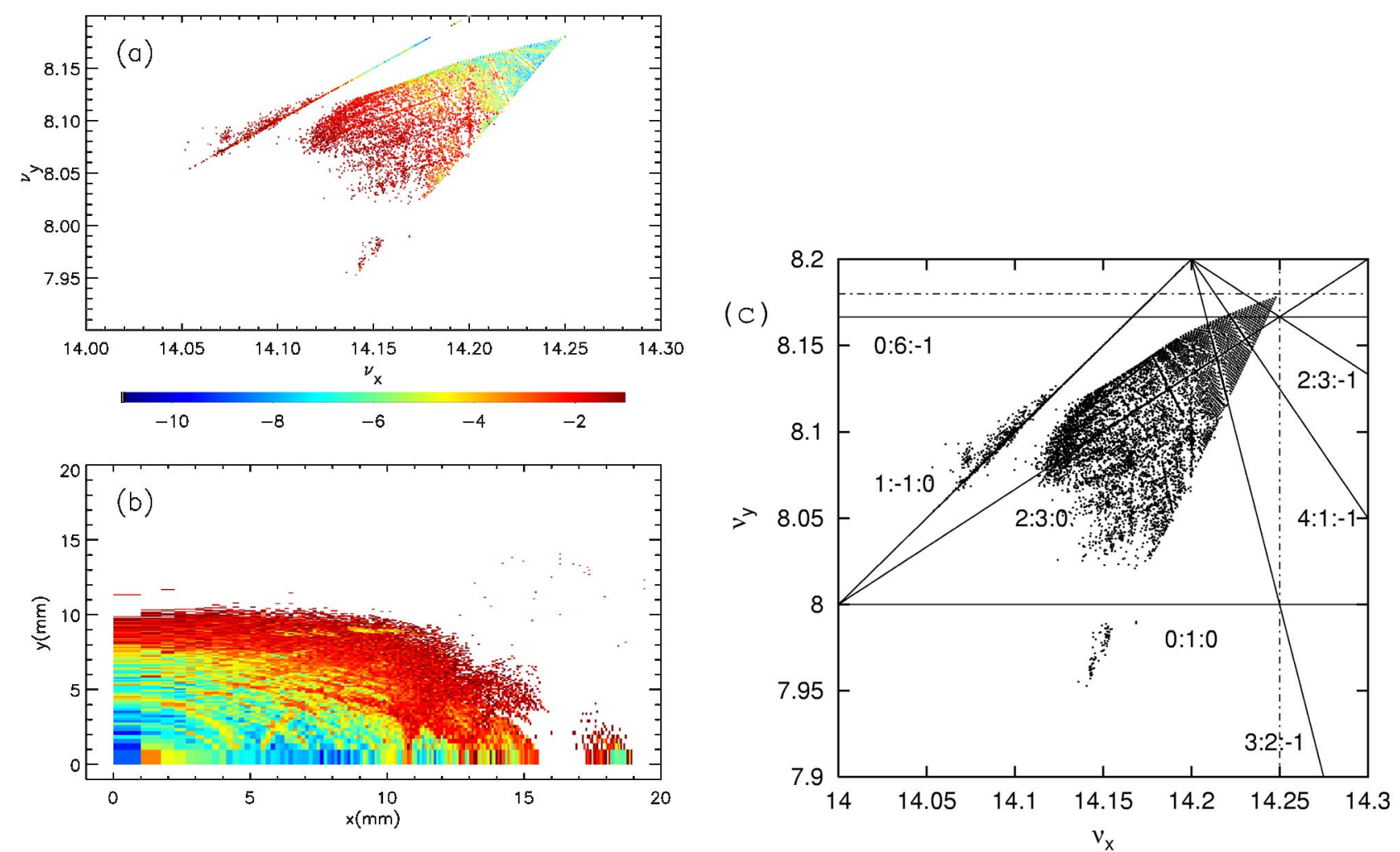

FIG. 17. (Color) ALS frequency map (a) and dynamic aperture (b) modeled with normal and skew quadrupole errors $\left(\beta_{x}=11.3 \mathrm{~m}\right.$ and $\beta_{y}=4.0 \mathrm{~m}$ ). The 12 -fold ring periodicity is broken; the map extension is reduced by a factor of 2 in the vertical plane. Fifthorder resonances not allowed by the ring periodicity are excited. Resonance network (c).

resonance $\nu_{y}-8=0$ is so excited by quadrupole errors that all regularity is destroyed in its vicinity.

The DA dimensions are now $[0,12]_{y=0} \times[0,8]_{x=0} \mathrm{~mm}$ since it is limited in the horizontal plane by the coupled resonance $\nu_{x}-2 \nu_{y}+2=0(1:-2: 0)$. The horizontal DA dimension is smaller than the physical aperture and very close to the value measured in the ring using a horizontal scraper. Therefore the FM is reduced by a factor of 2 in the vertical extent of the map. Resonances of 5th order are mainly excited $4 \nu_{x}+\nu_{y}-65=0$ $(4: 1:-1), \quad 3 \nu_{x}+2 \nu_{y}-59=0(3: 2:-1)$, and $2 \nu_{x}+$ $3 \nu_{y}-53=0(2: 3:-1)$ : they are unallowed resonances for the lattice [Fig. 17(c)]. On the whole the beam stability area is shrunk to the part around the working point.

The ALS is an important example of how small linear focusing errors can dramatically change the dynamics. The ideal map is not a good representation of the true dynamics. In order that the map accurately represents the beam dynamics requires knowing the linear field errors in the machine. This is true in all third generation light sources.

\section{Summary on ALS optics}

The ALS is a relatively simple machine. The FM presents no fold. There are only two sextupole families to correct the chromaticities. Yet in counterpart, for improving the dynamics only the working point may be moved. FMA enables us to get a clear insight of the beam dynamics. Main resonances are revealed, specially coupled resonances often not seen in the common lattice optimization scheme. Using the linear normal and skew magnetic default, the simulated dynamics is very close to the experimental measurements. Experiments were carried out at the ALS, where experimental frequency maps were measured for the first time. On momentum [24] and off momentum [4,27] were studies in detail with very good agreement between simulation and experiment.

\section{DISCUSSION}

Throughout this article, we gave an overview of the single particle transverse dynamics of a variety of storage rings: Super-ACO with its $72 \mathrm{~m}$ circumference, the ALS (196 m), SOLEIL (337 m), and the ESRF (844 m). For machine like Super-ACO, the so-called "small machine approximation" is no longer valid; in particular, the dipole and quadrupole fringe fields deeply impact the dynamics [28]. This is one of the reasons why we had to use two different tracking codes during this study. Using FMA as the main investigation tool, the beam dynamics is revealed in an easy and global manner. In that way, resonances limiting the DA and crossing resonance nodes are identified. Quantitative resonance widths are directly 
seen in FMs. The precision and fast convergence of FMA enables us to predict the beam dynamics for a modified working point (without additional computation). Shortly, we obtain an "identification card" or footprint of the beam dynamics.

The very different aspects of the frequency maps for, at first view rather similar, third generation light sources are surprising. Even for the same machine, small changes in the sextupolar strengths can produce significant modifications and distortions in the FMs. Apart from revealing the sensitivity of the dynamics to the change of parameters, the way the FM is folded is important.

For a typical machine, near the closed orbit, the Hamiltonian governing the dynamics is convex with respect to the action variables, which allows one to limit the diffusion of the trajectories. In particular, the diffusion will be limited by Nekhoroshev theorem bounds [29]. After the first fold that corresponds to the critical points of the frequency map, the Hamiltonian is no longer convex, and directions of fast escape may appear (see $[8,10]$ for more details). This is, we believe, an important point for the stability studies of particle beams that is well revealed by the study of the frequency maps, and we intend to analyze more precisely the diffusion of orbits in the case of a folded frequency map in future studies.

In general the dynamic apertures simulated and measured differ by at least a factor of 2 . Here we see that, once the experimental quadrupole defaults are known (obtained for instance from the orbit response matrix), a good understanding of the dynamics is reached in terms of injection efficiency, dynamics aperture, and resonances excited.

FMA can also be used to compute a FM directly on a running machine where the tracking code is substituted by turn by turn beam position monitors, and initial transverse coordinates are induced by two fast horizontal and vertical magnets. In this way, the method is model independent and allows comparison or matching with the machine model [24]. This method can then be incorporated in the control room of an accelerator as an on-line diagnostic tool. This is already the case at the ALS, and it can be expected that these experiments will be generalized to other machines [20].

\section{ACKNOWLEDGMENTS}

The authors are grateful to the ALS, ESRF, Super-ACO, and SOLEIL teams. L. N. thanks the Commissariat à l'Energie Atomique (Saclay, France) for a financial grant.

[1] P. Audy, Ph.D. thesis, Paris XI University, Orsay, 1989.

[2] J. Bengtsson, SLS internal Report No. SLS-TME-TA1997-0009, 1997.
[3] L. Nadolski, Ph.D. thesis, Paris XI University, CEA, DAPNIA/SEA-01-02-T, Orsay, 2001.

[4] C. Steier, D. Robin, Y. Wu, W. Decking, J. Laskar, and L. Nadolski, Phys. Rev. E, 65, 056506 (2002).

[5] J. Laskar, Icarus 88, 266-291 (1990).

[6] J. Laskar, Astron. Astrophys. 198, 341-362 (1988).

[7] J. Laskar, Physica (Amsterdam) 67D, 257-281 (1993).

[8] J. Laskar, in Proceedings of 3DHAM95, NATO Advanced Study Institutes (Kluwer Academic Publishers, Dordrecht, The Netherlands, 1999), pp. 134150.

[9] J. Laskar, Frequency Map Analysis and quasiperiodic decompositions, arxiv.org/abs/math.DS/0305364.

[10] J. Laskar, in Proceedings of the 20th Particle Accelerator Conference, Portland, 2003 (to be published).

[11] A. Morbidelli and A. Giorgilli, J. Stat. Phys. 78, 16071617 (1995).

[12] J. Laskar, in Workshop on Non-linear Dynamics in Particle Accelerators, Arcidosso-1994, edited by S. Chattopadhyay, M. Cornacchia, and C. Pellegrini, AIP Conf. No. 344 (AIP, New York, 1995), pp. 130-159.

[13] H. S. Dumas and J. Laskar, Phys. Rev. Lett. 70, 29752979 (1993).

[14] M. Belgroune, P. Brunelle, A. Nadji, and L. Nadolski, in Proceedings of the 20th Particle Accelerator Conference, Portland, 2003 (to be published).

[15] "SOLEIL: Rapport d'Avant Projet Détaillé," http:// www.synchrotron-soleil.fr/, CEA-CNRS.

[16] A. Nadji, P. Brunelle, J. M. Filhol, and M. P. Level, in Proceedings of 25th ICFA Advanced Beam Dynamics Workshop: Symposium on Intermediate-Energy Light Sources, Shanghai, 2001, edited by J. Corbett, Z. M. Dai, D. Einfeld, and Z.T. Zhao (SSRC, Shanghai, 2002), pp. 139-143.

[17] Tracking code based on a fourth-order Ruth integrator developed at the Advanced Light Source (ALS, Berkeley) by E. Forest.

[18] The ESRF Red Book, Grenoble, 1987, http:// www.esrf.fr/

[19] H. Grote and F. C. Iselin, The MAD Program, Version 8.1, User's Reference Manual, CERN Report No. CERN/SL/ 90-13, 1991.

[20] Y. Papaphilippou, L. Farvacques, A. Ropert, and J. Laskar, in Proceedings of the 20th Particle Accelerator Conference, Portland, 2003 (to be published).

[21] Super-ACO Conceptual Design Report, "Projet SuperACO,” No. LURE-IN2P2-CEA, 1981, http:// www.lure.u-psud.fr/

[22] 1-2 GeV Synchrotron Radiation Source Conceptual Design Report No. PUB-5172 Rev., 1986, http://wwwals.lbl.gov/

[23] J. Laskar and D. Robin, Part. Accel. 54, 183-192 (1996).

[24] D. Robin, C. Steier, J. Laskar, and L. Nadolski, Phys. Rev. Lett. 85, 558-560 (2000).

[25] D. Robin, J. Safranek, and W. Decking, Phys. Rev. ST Accel. Beams 2, 044001 (1999).

[26] J. Safranek, Nucl. Instrum. Methods Phys. Res., Sect. A 388, 27-36 (1997).

[27] C. Steier, D. Robin, Y. Wu, W. Decking, J. Laskar, and L. Nadolski, in Proceedings of the 19th Particle 
Accelerator Conference, Chicago, 2001 (IEEE, Piscataway, NJ, 2001), pp. 434-436.

[28] M. Belgroune, P. Brunelle, A. Nadji, and L. Nadolski, in Proceedings of the 8th European Particle Accelerator
Conference, Paris, 2002 (EPS-IGA, CERN, Geneva, 2002), pp. 1232-1234.

[29] N. N. Nekhoroshev, Russian Math. Surveys 32, 1-65 (1977). 